\title{
Fuzzy Non-Trivial Gauge Configurations
}

\author{
Badis Ydri \\ Physics Department, Syracuse University, \\ Syracuse, N.Y.,13244-1130, U.S.A.
}

\begin{abstract}
In this talk we will report on few results of discrete physics on the fuzzy sphere. In particular non-trivial field configurations such as monopoles and solitons are constructed on fuzzy $\mathbf{S}^{2}$ using the language of K-theory , i.e projectors . As we will show, these configurations are intrinsically finite dimensional matrix models . The corresponding monopole charges and soliton winding numbers are also found using the formalism of noncommutative geometry and cyclic cohomology .
\end{abstract}

Fuzzy physics is aimed to be an alternative method to approach discrete physics . Problems of lattice physics especially those with topological roots are all avoided on fuzzy spaces. For example, chiral anomaly, Fermion doubling and the discretization of non-trivial topological field configurations were all formulated consistently on the fuzzy sphere [see [1] and the extensive list of references therein]. The paradigm of fuzzy physics is "discretization by quantization ", namely given a space, we treat it as a phase space and then quantize it . This requires the existence of a symplectic structure on this space. One such class of spaces which admit symplectic forms are the co-adjoint orbits, for example $\mathbf{C P}^{1}=\mathbf{S}^{2}, \mathbf{C P}^{2}, \mathbf{C P}^{3}$ and so on . Their quantization to obtain their fuzzy counterparts is done explicitly in [2, 国] . Here we will only summarize the important results for $\mathbf{S}^{2}$ which are needed for the purpose of this paper .

\section{$1 \quad$ Fuzzy $\mathbf{S}^{2}$}

Fuzzy $\mathbf{S}^{2}$ or $\mathbf{S}_{F}^{2}$ is the algebra $\mathbf{A}=$ Mat $_{2 l+1}$ of $(2 l+1) \times(2 l+1)$ matrices which is generated by the operators $n_{i}^{F}, i=1,2,3$, which are defined by

$$
n_{i}^{F}=\frac{L_{i}}{\sqrt{l(l+1)}}
$$

$L_{i}$ 's satisfy $\left[L_{i}, L_{j}\right]=i \epsilon_{i j k} L_{k}$ and $\sum_{i=1}^{3} L_{i}^{2}=l(l+1)$ respectively, where $l$ is a positive integer. In other words, $L_{i}$ 's are the generators of the IRR $l$ of 
$S U(2)$. A general element $\hat{f}$ of $\mathbf{A}$ admits an expansion, in terms of $n_{i}^{F}$ 's , of the form $\hat{f}\left(\vec{n}^{F}\right)=\sum_{i_{1}, \ldots, i_{k}} f_{i_{1}, \ldots, i_{k}} n_{i_{1}}^{F} \ldots n_{i_{k}}^{F}$, which will terminate by the nature of the operators $n_{i}^{F}$ 's. The continuum limit is defined by $l \longrightarrow \infty$. In such a limit the fuzzy coordinates $n_{i}^{F}$ 's tend, by definition, to the commutative coordinates $n_{i}$ 's [ by inspection the commutators of the fuzzy coordinates among each others vanish at $l \longrightarrow \infty$, but from the Casimir equation above we must have $\left.\sum_{i=1}^{3} n_{i}^{2}=1\right]$. Furthermore, the noncommutative algebra at this limit becomes the commutative algebra of functions on continuum $\mathbf{S}^{2}$, namely $\mathrm{A} \longrightarrow \mathcal{A}$, where a general element $f$ of $\mathcal{A}$ will admit the expansion $f(\vec{n})=\sum_{i_{1}, \ldots, i_{k}} f_{i_{1}, \ldots, i_{k}} n_{i_{1} \ldots .} n_{i_{k}}$.

Viewing $\mathbf{S}^{2}$ as a submanifold of $\mathbf{R}^{3}$, one can check the following basic identity 3]

$$
\mathcal{D}_{2}=\left.\mathcal{D}_{3}\right|_{r=\rho}+\frac{i \gamma^{3}}{\rho} .
$$

$\gamma^{a}=\sigma_{a}, a=1,2,3$, are the flat gamma matrices in 3-dimensions $. \mathcal{D}_{2}, \mathcal{D}_{3}$ are the Dirac operators on $\mathbf{S}^{2}$ and $\mathbf{R}^{3}$ respectively.$\left.\mathcal{D}_{3}\right|_{r=\rho}$ is the restriction of the Dirac operator on $\mathbf{R}^{3}$ to the sphere $r=\rho$, where $\rho$ is the radius of the sphere, namely $\sum_{a=1}^{3} x_{a}^{2}=\rho^{2}$ for any $\vec{x} \in \mathbf{S}^{2}$. The Clifford algebra on $\mathbf{S}^{2}$ is two dimensional and therefore at each point $\vec{n}=\vec{x} / \rho$ one has only two independents gamma matrices, they can be taken to be $\gamma^{1}$ and $\gamma^{2} \cdot \gamma^{3}$ should then be identified with the chirality operator $\gamma=\vec{\sigma} \cdot \vec{n}$ on $\mathbf{S}^{2}$.

Next by using the canonical Dirac operator $\mathcal{D}_{3}=-i \sigma_{a} \partial_{a}$ in (2) one can derive the two following equivalent expressions for the Dirac operator $\mathcal{D}_{2}$ on $\mathbf{S}_{2}$ :

$$
\begin{aligned}
\mathcal{D}_{2 g} & =\frac{1}{\rho}(\vec{\sigma} \overrightarrow{\mathcal{L}}+1) \\
\mathcal{D}_{2 w} & =-\frac{1}{\rho} \epsilon_{i j k} \sigma_{i} n_{j} \mathcal{J}_{k} .
\end{aligned}
$$

$\mathcal{L}_{k}=-i \epsilon_{k i j} x_{i} \partial_{j}$ is the orbital angular momentum and $\mathcal{J}_{k}=\mathcal{L}_{k}+\frac{\sigma_{k}}{2}$ is the total angular momentum . $g$ and $w$ in (3) stands for Grosse-KlimčíkPrešnajder [3] and Watamuras Dirac operators [4] respectively . It is not difficult to check that $\mathcal{D}_{2 w}=i \gamma \mathcal{D}_{2 g}=\left.\mathcal{D}_{3}\right|_{r=\rho}+\frac{i \gamma}{\rho}$ which means that $\mathcal{D}_{2 w}$ and $\mathcal{D}_{2 g}$ are related by a unitary transformation and therefore are equivalent. The spectrum of these Dirac operators is trivially derived to be given by $\pm \frac{1}{\rho}\left(j+\frac{1}{2}\right)$ where $j$ is the eigenvalue of $\overrightarrow{\mathcal{J}}$, i.e $\overrightarrow{\mathcal{J}}^{2}=j(j+1)$ and $j=1 / 2,3 / 2, \ldots$. 
The fuzzy versions of the Dirac operators (3) are taken to be

$$
\begin{aligned}
D_{2 g} & =\frac{1}{\rho}(\vec{\sigma} \cdot a d \vec{L}+1) \\
D_{2 w} & =\frac{1}{\rho} \epsilon_{i j k} \sigma_{i} n_{j}^{F} L_{k}^{R} .
\end{aligned}
$$

$a d \vec{L}=\vec{L}^{L}-\vec{L}^{R}$ is the fuzzy derivation which annihilates the identity matrix in $\mathbf{A}$ as the classical derivation $\overrightarrow{\mathcal{L}}$ annihilates the constant function in $\mathcal{A} . \vec{L}^{L}$ and $-\vec{L}^{R}$ are the generators of the IRR $l$ of $S U(2)$ which act on the left and on the right of the algebra $\mathbf{A}$ respectively, i.e $\vec{L}^{L} f=\vec{L} f$ and $-L_{i}^{R} f=-f L_{i}$ for any $f \in \mathbf{A}$. From this definition one can see that $A d L_{i}$ provide the generators of the adjoint action of $S U(2)$ on $\mathbf{A}$, namely $A d \vec{L}(f)=[\vec{L}, f]$ for any $f \in \mathbf{A}$.

These two fuzzy Dirac operators are not unitarily equivalent anymore . This can be checked by computing their spectra. The spectrum of $D_{2 g}$ is exactly that of the continuum only cut-off at the top total angular momentum $j=2 l+\frac{1}{2}$. In other words the spectrum of $D_{2 g}$ is equal to $\left\{ \pm \frac{1}{\rho}\left(j+\frac{1}{2}\right), j=\right.$ $\left.\frac{1}{2}, \frac{3}{2}, \ldots 2 l-\frac{1}{2}\right\}$ and $D_{2 g}(j)=\frac{1}{\rho}\left(j+\frac{1}{2}\right)$ for $j=2 l+\frac{1}{2}$. The spectrum of $D_{2 w}$ is , however , highly deformed as compared to the continuum spectrum especially for large values of $j$. It is given by $D_{2 w}(j)= \pm \frac{1}{\rho}\left(j+\frac{1}{2}\right) \sqrt{1+\frac{1-(j+1 / 2)^{2}}{4 l(l+1)}}$. From these results it is obvious that $D_{2 g}$ is superior to $D_{2 w}$ as an approximation to the continuum .

In the same way one can find the fuzzy chirality operator $\Gamma$ by the simple replacement $\vec{n} \longrightarrow \vec{n}^{F}$ in $\gamma=\vec{\sigma} \cdot \vec{n}$ and insisting on the result to have the following properties : 1$) \Gamma^{2}=1, \Gamma^{+}=\Gamma$ and $[\Gamma, f]=0$ for all $f \in \mathbf{A}$. One then finds $[4$

$$
\Gamma=\frac{1}{l+\frac{1}{2}}\left(-\vec{\sigma} \vec{L}^{R}+\frac{1}{2}\right) .
$$

Interestingly enough this fuzzy chirality operator anticommutes with $D_{2 w}$ and not with $D_{2 g}$ so $D_{2 w}$ is a better approximation to the continuum than $D_{2 g}$ from this respect. This is also clear from the spectra above, in the spectrum of $D_{2 g}$ the top angular momentum is not paired to anything and therefore $D_{2 g}$ does not admit a chirality operator .

\section{Fuzzy Non-Trivial Gauge Configurations}




\subsection{Classical Monopoles}

Monopoles are one of the most fundamental non trivial configurations in field theory. The wave functions of a particle of charge $q$ in the field of a monopole $p$, which is at rest at $r=0$, are known to be given by the expansion [2]

$$
\psi^{(N)}(r, g)=\sum_{j, m} c_{m}^{j}(r)<j, m\left|D^{(j)}(g)\right| j,-\frac{N}{2}>
$$

where $D^{(j)}: g \longrightarrow D^{(j)}(g)$ is the $j$ IRR of $g \in S U(2)$. The integer $N$ is related to $q$ and $p$ by the Dirac quantization condition : $N=\frac{q p}{2 \pi} \cdot r$ is the radial coordinate of the relative position $\vec{x}$ of the system, the angular variables of $\vec{x}$ are defined through the element $g \in S U(2)$ by $\vec{\tau} \cdot \vec{n}=g \tau_{3} g^{-1}, \vec{n}=\vec{x} / r$. It is also a known result that the precise mathematical structure underlying this physical system is that of a $U(1)$ principal fiber bundle $S U(2) \longrightarrow \mathbf{S}^{2}$. In other words for a fixed $r=\rho$, the particle $q$ moves on a sphere $\mathbf{S}^{2}$ and its wave functions (6) are precisely elements of $\mathcal{S}\left(\mathbf{S}^{2}, S U(2)\right)$, namely sections of a $U(1)$ bundle over $\mathbf{S}^{2}$. They have the equivariance property

$$
\psi^{(N)}\left(\rho, g e^{i \theta \frac{\tau_{3}}{2}}\right)=e^{-i \theta \frac{N}{2}} \psi^{(N)}(\rho, g),
$$

i.e they are not really functions on $\mathbf{S}^{2}$ but rather functions on $S U(2)$ because they clearly depend on the specific point on the $U(1)$ fiber . In this paper , we will only consider the case $N= \pm 1$. The case $|N| \neq 1$ being similar and is treated in great detail in [1, 5].

An alternative description of monopoles can be given in terms of $\mathrm{K}$ theory and projective modules. It is based on the Serre-Swan's theorem [7, 8]which states that there is a complete equivalence between vector bundles over a compact manifold $\mathbf{M}$ and projective modules over the algebra $C(\mathbf{M})$ of smooth functions on $\mathbf{M}$. Projective modules are constructed from $C(\mathbf{M})^{\mathbf{n}}=\mathbf{C}(\mathbf{M}) \otimes \mathbf{C}^{\mathbf{n}}$ where $n$ is some integer by the application of a certain projector $p$ in $\mathcal{M}_{n}(C(\mathbf{M}))$, i.e the algebra of $n \times n$ matrices with entries in $C(\mathbf{M})$.

In our case $\mathbf{M}=\mathbf{S}^{2}$ and $C(\mathbf{M})=\mathcal{A} \equiv$ the algebra of smooth functions on $\mathrm{S}^{2}$. For a monopole system with winding number $N= \pm 1$, the appropriate projective module will be constructed from $\mathcal{A}^{2}=\mathcal{A} \otimes \mathbf{C}^{2}$. It is $\mathcal{P}^{( \pm 1)} \mathcal{A}^{2}$ where $\mathcal{P}^{( \pm 1)}$ is the projector

$$
\mathcal{P}^{( \pm 1)}=\frac{1 \pm \vec{\tau} \cdot \vec{n}}{2}
$$


It is clearly an element of $\mathcal{M}_{2}(\mathcal{A})$ and satisfies $\mathcal{P}^{( \pm 1) 2}=\mathcal{P}^{( \pm 1)}$ and $\mathcal{P}^{( \pm 1)+}=$ $\mathcal{P}^{( \pm 1)}$. $\mathcal{P}^{( \pm 1)} \mathcal{A}^{2}$ describes a monopole system with $N= \pm 1$ as one can directly check by computing its Chern character as follows

$$
\pm 1=\frac{1}{2 \pi i} \int \operatorname{Tr} \mathcal{P}^{( \pm 1)} d \mathcal{P}^{( \pm 1)} \wedge d \mathcal{P}^{( \pm 1)}
$$

On the contrary to the space of sections $\mathcal{S}\left(\mathbf{S}^{2}, S U(2)\right)$, elements of $\mathcal{P}^{( \pm 1)} \mathcal{A}^{2}$ are by construction invariant under the action $g \longrightarrow g \exp \left(i \theta \frac{\tau_{3}}{2}\right)$. The other advantage of $\mathcal{P}^{( \pm 1)} \mathcal{A}^{2}$ as compared to $\mathcal{S}\left(\mathbf{S}^{2}, S U(2)\right)$ is the fact that its fuzzification is much more straight forward.

\subsection{On The Equivalence of $\mathcal{P}^{( \pm 1)} \mathcal{A}^{2}$ and $\mathcal{S}\left(\mathbf{S}^{2}, S U(2)\right)$}

Before we start the fuzzification of $\mathcal{P}^{( \pm 1)} \mathcal{A}^{2}$, let us first comment on the relation between the wave functions $\psi^{( \pm 1)}$ given in equation (6) and those belonging to $\mathcal{P}^{( \pm 1)} \mathcal{A}^{2}$. The projector $\mathcal{P}^{( \pm 1)}$ can be rewritten as $\mathcal{P}^{( \pm 1)}=$ $D^{\left(\frac{1}{2}\right)} \frac{1 \pm \tau_{3}}{2} D^{\left(\frac{1}{2}\right)+}(g)$ where $D^{\left(\frac{1}{2}\right)}: g \longrightarrow D^{\left(\frac{1}{2}\right)}(g)=g$ is the $\frac{1}{2}$ IRR of $S U(2)$. Hence $\mathcal{P}^{( \pm 1)} D^{\left(\frac{1}{2}\right)}(g)\left| \pm>=D^{\left(\frac{1}{2}\right)}(g) \frac{1 \pm \tau_{3}}{2}\right| \pm>=D^{\left(\frac{1}{2}\right)}(g) \mid \pm>$, where $\mid \pm>$ are defined by $\tau_{3}| \pm>= \pm| \pm>$. In the same way one can show that $\mathcal{P}^{( \pm 1)} D^{\left(\frac{1}{2}\right)}(g) \mid \mp>=0$. This last result means that

$$
\mathcal{P}^{( \pm 1)}=D^{\left(\frac{1}{2}\right)}(g)| \pm>< \pm| D^{\left(\frac{1}{2}\right)+}(g)
$$

$< \pm \mid D^{\left(\frac{1}{2}\right)+}(g)$ defines a map from $\mathcal{P}^{( \pm 1)} \mathcal{A}^{2}$ into $\mathcal{S}\left(\mathbf{S}^{2}, S U(2)\right)$ as follows

$$
< \pm\left|D^{\left(\frac{1}{2}\right)+}(g) \quad: \quad\right| \psi>\longrightarrow< \pm\left|D^{\left(\frac{1}{2}\right)+}(g)\right| \psi>=\psi^{( \pm 1)}(\rho, g) .
$$

$< \pm\left|D^{\left(\frac{1}{2}\right)+}(g)\right| \psi>$ has the correct transformation law (17) under $g \longrightarrow g \exp \left(i \theta \frac{\tau_{3}}{2}\right)$ as one can check by using the basic equivariance property

$$
D^{\left(\frac{1}{2}\right)}\left(g e^{i \theta \frac{\tau_{3}}{2}}\right)\left| \pm>=e^{ \pm i \frac{\theta}{2}} D^{\left(\frac{1}{2}\right)}(g)\right| \pm>.
$$

In the same way $D^{\left(\frac{1}{2}\right)}(g) \mid \pm>$ defines a map, $\mathcal{S}\left(\mathbf{S}^{2}, S U(2)\right) \longrightarrow \mathcal{P}^{( \pm 1)} \mathcal{A}^{2}$, which takes the wave functions $\psi^{( \pm 1)}$ to the two components elements $\psi^{( \pm 1)} D^{\left(\frac{1}{2}\right)}(g) \mid \pm>$ of $\mathcal{P}^{( \pm 1)} \mathcal{A}^{2}$. Under $g \longrightarrow g \exp \left(i \theta \frac{\tau_{3}}{2}\right)$, the two phases coming from $\psi^{( \pm 1)}$ and $D^{\left(\frac{1}{2}\right)}(g) \mid \pm>$ cancel exactly so that their product is a function over $\mathbf{S}^{2}$. 


\subsection{Fuzzy Monopoles}

Towards fuzzification one rewrites the winding number (9) in the form

$$
\begin{aligned}
\pm 1 & =-\frac{1}{4 \pi} \int d(\cos \theta) \wedge d \phi \operatorname{Tr} \gamma \mathcal{P}^{( \pm 1)}\left[\mathcal{D}, \mathcal{P}^{( \pm 1)}\right]\left[\mathcal{D}, \mathcal{P}^{( \pm 1)}\right](\vec{n}) \\
& =-\operatorname{Tr}_{\omega}\left(\frac{1}{|\mathcal{D}|^{2}} \gamma \mathcal{P}^{( \pm 1)}\left[\mathcal{D}, \mathcal{P}^{( \pm 1)}\right]\left[\mathcal{D}, \mathcal{P}^{( \pm 1)}\right]\right)
\end{aligned}
$$

The first line is trivial to show starting from (9), whereas the second line is essentially Connes trace theorem [7] . $|\mathcal{D}|=$ positive square root of $\mathcal{D}^{\dagger} \mathcal{D}$ while $\operatorname{Tr}_{\omega}$ is the Dixmier trace [7, 9, 10].In the fuzzy setting, this Dixmier trace will be replaced by the ordinary trace because the algebra of functions on fuzzy $\mathbf{S}_{F}^{2}$ is finite dimensional .

$\mathcal{D}$ in (13) is either $\mathcal{D}_{2 g}$ or $\mathcal{D}_{2 w}$ which are given in equation (3) . They both give the same answer \pm 1 . The fuzzy analogues of $\mathcal{D}_{2 g}$ and $\mathcal{D}_{2 w}$ are respectively $D_{2 g}$ and $D_{2 w}$ given by equation (雨) . These latter operators were shown to be different and therefore one has to decide which one should we take as our fuzzy Dirac operator . $D_{2 g}$ does not admit as it stands a chirality operator and therefore its use in the computation of winding numbers requires more care which is done in [1, 6].$D_{2 w}$ admits the fuzzy chirality operator (5) which will be used instead of the continuum chirality $\gamma=\vec{\sigma} \cdot \vec{n}$. However $D_{2 w}$ has a zero eigenvalue for $j=2 l+\frac{1}{2}$ so it must be regularized for its inverse in (13) to make sense. This will be understood but not done explicitly in this paper, a careful treatment is given in [1, 5].

Finally the projector $\mathcal{P}^{( \pm 1)}$ will be replaced by a fuzzy projector $p^{( \pm 1)}$ which we will now find. We proceed like we did in finding the chirality operator $\Gamma$, we replace $\vec{n}$ in (8) by $\vec{n}^{F}=\vec{L}^{L} / \sqrt{l(l+1)}$ and insist on the result to have the properties $p^{( \pm 1) 2}=p^{( \pm 1)}$ and $p^{( \pm 1)+}=p^{( \pm 1)}$. We also require this projector to commute with the chirality operator $\Gamma$, the answer for winding number $N=+1$ turns out to be $p^{(+1)}=\frac{1}{2}+\frac{1}{2 l+1}\left[\vec{\tau} \cdot \vec{L}^{L}+\frac{1}{2}\right]$. This can be rewritten in the following useful form

$$
p^{(+1)}=\frac{\vec{K}^{(1) 2}-\left(l-\frac{1}{2}\right)\left(l+\frac{1}{2}\right)}{\left(l+\frac{1}{2}\right)\left(l+\frac{3}{2}\right)-\left(l-\frac{1}{2}\right)\left(l+\frac{1}{2}\right)},
$$

where $\vec{K}^{(1)}=\vec{L}^{L}+\frac{\vec{\tau}}{2}$. This allows us to see immediately that $p^{(+1)}$ is the projector on the subspace with the maximum eigenvalue $l+\frac{1}{2}$. Similarly, 
the projector $p^{(-1)}$ will correspond to the subspace with minimum eigenvalue $l-\frac{l}{2}$, namely

$$
p^{(-1)}=\frac{\vec{K}^{(1) 2}-\left(l+\frac{1}{2}\right)\left(l+\frac{3}{2}\right)}{\left(l-\frac{1}{2}\right)\left(l+\frac{1}{2}\right)-\left(l+\frac{1}{2}\right)\left(l+\frac{3}{2}\right)} .
$$

By construction (14) as well as (15) have the correct continuum limit (8), and they are in the algebra $\mathcal{M}_{2}(\mathbf{A})$ where $\mathbf{A}$ is the fuzzy algebra on fuzzy $\mathbf{S}_{F}^{2}$, i.e $2(2 l+1) \times 2(2 l+1)$ matrices . Fuzzy monopoles with winding number \pm 1 are then described by the projective modules $p^{( \pm 1)} \mathbf{A}^{2}$.

If one include spin, then $\mathbf{A}^{2}$ should be enlarged to $\mathbf{A}^{4}$. It is on this space that the Dirac operator $D_{2 w}$ as well as the chirality operator $\Gamma$ are acting. In the fuzzy the left and right actions of the algebra $\mathbf{A}$ on $\mathbf{A}$ are not the same. The left action is generated by $L_{i}^{L}$ whereas the right action is generated by $-L_{i}^{R}$ so that we are effectively working with the algebra $\mathbf{A}^{L} \otimes \mathbf{A}^{R}$. A representation $\Pi$ of this algebra is provided by $\Pi(\alpha)=\alpha \otimes \mathbf{1}_{2 \times 2}$ for any $\alpha \in \mathbf{A}^{L} \otimes \mathbf{A}^{R}$. It acts on the Hilbert space $\mathbf{A}^{4} \oplus \mathbf{A}^{4}$.

With all these considerations, one might as well think that one must naively replace $\operatorname{Tr}_{\omega} \longrightarrow \operatorname{Tr}, \gamma \longrightarrow \Gamma, \mathcal{D} \longrightarrow D_{2 w}$ and $\mathcal{P}^{( \pm 1)} \longrightarrow p^{( \pm 1)}$ in (13) to get its fuzzy version. This is not totally correct since the correct discrete version of (13) turns out to be

$$
c( \pm 1)=-\operatorname{Tr} \epsilon P^{( \pm 1)}\left[F_{2 w}, P^{( \pm 1)}\right]\left[F_{2 w}, P^{( \pm 1)}\right]
$$

with

$$
\mathbf{F}_{2 w}=\left(\begin{array}{cc}
0 & \frac{D_{2 w}}{\left|D_{2 w}\right|} \\
\frac{D_{2 w}}{\left|D_{2 w}\right|} & 0
\end{array}\right), \quad \epsilon=\left(\begin{array}{cc}
\Gamma & 0 \\
0 & \Gamma
\end{array}\right)
$$

and

$$
P^{( \pm 1)}=\left(\begin{array}{cc}
\frac{1+\Gamma}{2} p^{( \pm)} & 0 \\
0 & \frac{1-\Gamma}{2} p^{( \pm)}
\end{array}\right) .
$$

[For a complete proof see [1] or [5]].For $p^{(+1)}$ one finds that $c(+1)=+1+$ $[2(2 l+1)+1]$ while for $p^{(-)}$we find $c(-1)=-1+[2(2 l)+1]$. They are both wrong if compared to (13)!

The correct answer is obtained by recognizing that $c( \pm 1)$ is nothing but the index of the operator

$$
\hat{f}^{(+)}=\frac{1-\Gamma}{2} p^{( \pm 1)} \frac{D_{2 w}}{\left|D_{2 w}\right|} p^{( \pm 1)} \frac{1+\Gamma}{2} .
$$


This index counts the number of zero modes of $\hat{f}^{(+)}$. The proof starts by remarking that, by construction, only the matrix elements $<p^{( \pm 1)} U_{-}\left|\hat{f}^{(+)}\right| p^{( \pm 1)} U_{+}>$ where $U_{ \pm}=\frac{1 \pm \Gamma}{2} \mathbf{A}^{4}$, exist and therefore $\hat{f}^{(+)}$is a mapping from $\hat{V}_{+}=p^{( \pm 1)} U_{+}$ to $\hat{V}_{-}=p^{( \pm 1)} U_{-}$. Hence Index $\hat{f}^{(+)}=\operatorname{dim} \hat{V}_{+}-\operatorname{dim} \hat{V}_{-}$.

Since one can write the chirality operator $\Gamma$ in the form $\Gamma=\frac{1}{l+1 / 2}[j(j+$ $\left.1)-(l+1 / 2)^{2}\right]$ where $j$ is the eigenvalue of $\left(-\vec{L}^{R}+\frac{\vec{\sigma}}{2}\right)^{2}, j=l \pm 1 / 2$ for which $\left.\Gamma\right|_{j=l \pm 1 / 2}= \pm 1$ defines the subspace $U_{ \pm}$with dimension $2(l \pm 1 / 2)+1$. On the other hand, for $p^{(+1)}$ which projects down to the subspace with maximum eigenvalue $k_{\max }=l+\frac{1}{2}$ of the operator $\vec{K}^{(1)}=\vec{L}+\frac{\vec{\tau}}{2}, \hat{V}_{ \pm}$has dimension $[2(l \pm 1 / 2)+1][2(l+1 / 2)+1]$ and so the index is $\operatorname{Index} \hat{f}^{(+)}=c(+1)=2(2 l+2)$. This result signals the existence of zero modes of the operator $\hat{f}^{(+)}$. Indeed for $\Gamma=+1$ one must couple $l+\frac{1}{2}$ to $l+\frac{1}{2}$ and obtain $j=2 l+1,2 l, . .0$, whereas for $\Gamma=-1$ we couple $l+\frac{1}{2}$ to $l-\frac{1}{2}$ and obtain $j=2 l, \ldots, 1 . j$ here denotes the total angular momentum $\vec{J}=\vec{L}^{L}-\vec{L}^{R}+\frac{\vec{\sigma}}{2}+\frac{\vec{\tau}}{2}$. Clearly the eigenvalues $j^{(+1)}=2 l+1$ and 0 in $\hat{V}_{+}$are not paired to anything. The extra piece in $c(+1)$ is therefore exactly equal to the number of the top zero modes , namely $2 j^{(+1)}+1=2(2 l+1)+1$. These modes do not exist in the continuum and therefore they are of no physical relevance and must be projected out . This can be achieved by replacing the projector $p^{(+1)}$ by a corrected projector $\pi^{(+1)}=p^{(+1)}\left[1-\pi^{\left(j^{(+1)}\right)}\right]$ where $\pi^{\left(j^{(+1)}\right)}$ projects out the top eigenvalue $j^{(+1)}$, it can be easily written down explicitly . Putting $\pi^{(+1)}$ in (16) gives exactly $c(+1)=+1$ which is the correct answer .

The same analysis goes for $p^{(-1)}$. Indeed if we replace it by the corrected projector $\pi^{(-1)}=p^{(-1)}\left[1-\pi^{\left(j^{(-1)}\right)}\right]$ where $\pi^{\left(j^{(-1)}\right)}$ projects out the top eigenvalue $j^{(-1)}=2 l$, then equation (16) will give exactly $c(-1)=-1$ which is what we want .

\section{Conclusion}

It was shown in this article that topological quantities can be precisely and strictly defined in the discrete setting by using the methods of noncommutative geometry and fuzzy physics .

\section{References}


[1] Badis Ydri , Fuzzy Physics, a thesis which will be submitted in partial fulfillment of the requirements for the degree of Ph.D in physics, syracuse university .

[2] A.P.Balachandran , G.Marmo ,B-S.Skagerstan and A.Stern, Classical Topology and Quantum States, World Scientific, Singapore, 1991.

[3] H. Grosse and P. Prešnajder, Lett.Math.Phys. 33, 171 (1995). H. Grosse, C. Klimčík and P. Prešnajder,Commun.Math.Phys. 178,507-526 (1996); 185, 155 (1997);H. Grosse and P. Prešnajder, Lett.Math.Phys. 46, 61 (1998) and ESI preprint,1999. H. Grosse, C. Klimčík, and P. Prešnajder,Comm.Math.Phys. 180, 429 (1996),hep-th/9602115.H. Grosse, C. Klimčik, and P. Prešnajder, in Les Houches Summer School on Theoretical Physics, 1995,hep-th/9603071.

[4] U. Carow-Watamura and S. Watamura,hep-th/9605003, Comm.Math.Phys. 183, 365 (1997), hep-th/9801195.

[5] S. Baez, A. P. Balachandran, S. Vaidya and B. Ydri,hep-th/9811169 and Comm.Math.Phys.208,787(2000).

[6] A. P. Balachandran, T. R. Govindarajan and B. Ydri, $S U$ 4240-712,IMSc-99/10/36 and hep-th/9911087;A. P. Balachandran, T. R. Govindarajan and B. Ydri, SU-4240-712,IMSc-99/10/36 and hep-th/0006216 and Mod.Phys.Lett.A15, 1279 (2000);

[7] A. Connes, Noncommutative Geometry,Academic Press, London, 1994;

[8] G. Landi ."Deconstructing Monopoles and Instantons" . math-ph/9812004.

[9] G. Landi, An Introduction to Noncommutative spaces And Their Geometries, Springer-Verlag, Berlin, 1997.hep-th/9701078.

[10] J. C. Varilly and J. M. Gracia-Bondia. J. Geom. Phys., 12:223301, 1993.J. C. Varilly. An Introduction to Noncommutative Geometry. physics $/ 9709045$. 